\title{
Approximate multipliers and approximate double centralizers: A fixed point approach
}

\author{
Abasalt Bodaghi, Madjid Eshaghi Gordji and Kamal Paykan
}

\begin{abstract}
In the present paper, the Hyers-Ulam stability and also the superstability of double centralizers and multipliers on Banach algebras are established by using a fixed point method. With this method, the condition of without order on Banach algebras is no longer necessary.
\end{abstract}

\section{Introduction}

The concept of the stability and the superstability for Banach algebra has been a main stream in the theory of Banach algebras in the last decades. A functional equation is called stable if any approximately solution to the functional equation is near to a true solution of that functional equation, and is superstable if every approximately solution is an exact solution of it.

In 1940, Ulam [21] proposed the following question concerning the stability of group homomorphisms: under what condition does there exist an additive mapping near an approximately additive mapping? Hyers [13] answered the problem of Ulam for the case where $X$ and $Y$ are Banach spaces. A generalized version of the theorem of Hyers for approximately linear mapping was given by Th. M. Rassias [19]. Since then, the stability problems of various functional equation have been extensively investigated by a number of authors (for instances, [6], [7], [10], and [14]).

Key Words: Banach algebra; Double centralizer; Hyers-Ulam stability; Multiplier; Superstability.

2010 Mathematics Subject Classification: Primary 39B82; Secondary 39B52, 47H10, 39B82, 46H 25 .

Received: April, 2011

Revised: April, 2011.

Accepted: February, 2012. 
In 2003, Cădariu and Radu [3] applied the fixed point method to the investigation of the Jensen functional equation (see $[2,4,8,9]$ for more applications of this method). They presented a short and a simple proof (different from the "direct method", initiated by Hyers in 1941) for the Hyers-Ulam stability of the Jensen functional equation [18], for the Cauchy functional equation [4] and for the quadratic functional equation [3].

Let $\mathcal{A}$ be a non-unital Banach algebra. Then $\mathcal{A}^{\#}=\mathcal{A} \oplus \mathbb{C}$ is a unital Banach algebra such that $\mathcal{A}$ is a closed subalgebra of $\mathcal{A}^{\#}$. In fact $\mathcal{A}^{\#}$ is the smallest unitization of $\mathcal{A}$. Also there are other unitizations for Banach algebras. For instance, the multiplier of $\mathcal{A}, \mathcal{M}(\mathcal{A})$ is one of them. However, $\mathcal{M}(\mathcal{A})$ is very much bigger than $\mathcal{A}^{\#}$.

The concept of the multipliers of Banach algebras were defined by Helgason in [11]. Later, Wang in [22] studied the multipliers on commutative Banach algebras. For some non-unital Banach algebras, their multipliers are computed. If $X$ is a locally compact Housdorff space, then $\mathcal{M}\left(C_{0}(X)\right)=C_{b}(X)$, where $C_{0}(X)$ is Banach algebra $\left(C^{*}\right.$-algebra) of continuous functions on $X$ which vanish at infinity, and $C_{b}(X)$ is Banach algebra of all bounded continuous complex-valued functions on $X$. For Hilbert space $\mathcal{H}$, the multiplier of the compact operators on $\mathcal{H}$ is the bounded operators on $\mathcal{H}$.

Let $\mathcal{A}$ be an algebra. Recall that $A_{l}(\mathcal{A}):=\{a \in \mathcal{A}: a \mathcal{A}=\{0\}\}$ is the left annihilator ideal and $A_{r}(\mathcal{A}):=\{a \in \mathcal{A}: \mathcal{A} a=\{0\}\}$ is the right annihilator ideal on $\mathcal{A}$. We say a Banach algebra $\mathcal{A}$ is (strongly) without order if $A_{l}(\mathcal{A})=A_{r}(\mathcal{A})=\{0\}$. Obviously, a Banach algebra is strongly without order when $\mathcal{A}$ is unital or approximately unital.

Miura, Hirasawa and Takasaki in [16, Theorem 1.3] investigated the stability of multipliers on Banach algebras, and showed that every approximately multiplier on a Banach algebra can be approximated by a multiplier. They also proved the superstability multipliers with the condition of without order on Banach algebras. On the other hand, the notion of double centralizer was introduced by Hochschild [12] and Johnson [15] independently. The stability and the superstability of double centralizers of a Banach algebra $\mathcal{A}$ which is (strongly) without order is investigated in [17].

In this paper, we remove the condition of without order on Banach algebras. In other words, we show that the hypothesis on Banach algebras being without order in $[16,17]$ can be eliminated, and establish the stability and the superstability of double centralizers and multipliers on a Banach algebra by a method of the fixed point. 


\section{Stability of double centralizers}

Before proceeding to the main results, we will state the following theorem which is useful to our purpose (an extension of the result was given in [20]).

Theorem 2.1. (The fixed point alternative $[5])$ Let $(\Omega, d)$ be a complete generalized metric space and $\mathcal{J}: \Omega \rightarrow \Omega$ be a mapping with Lipschitz constant $L<1$. Then, for each element $x \in \Omega$, either $d\left(g^{n} x, g^{n+1} x\right)=\infty$ for all $n \geq 0$, or there exists a natural number $n_{0}$ such that:

(i) $d\left(g^{n} x, g^{n+1} x\right)<\infty$ for all $n \geq n_{0}$;

(ii) the sequence $\left\{\mathcal{J}^{n} x\right\}$ is convergent to a fixed point $y^{*}$ of $\mathcal{J}$;

(iii) $y^{*}$ is the unique fixed point of $\mathcal{J}$ in the set

$$
\Lambda=\left\{y \in \Omega: d\left(g^{n_{0}} x, y\right)<\infty\right\}
$$

(iv) $d\left(y, y^{*}\right) \leq \frac{1}{1-L} d(y, \mathcal{J} y)$ for all $y \in \Lambda$.

Throughout this paper, we assume that $A$ is a complex Banach algebra and denote $\overbrace{A \times A \times \ldots \times A}^{n \text {-times }}$ by $A^{n}$. A linear mapping $L: A \longrightarrow A$ is said to be left centralizer on $A$ if $L(a b)=L(a) b$ for all $a, b \in A$. Similarly, a linear mapping $R: A \longrightarrow A$ satisfying $R(a b)=a R(b)$ for all $a, b \in A$ is called right centralizer on $A$. A double centralizer on $A$ is a pair $(L, R)$, where $L$ is a left centralizer, $R$ is a right centralizer and $a L(b)=R(a) b$ for all $a, b \in A$. For example, $\left(L_{c}, R_{c}\right)$ is a double centralizer, where $L_{c}(a):=c a$ and $R_{c}(a):=a c$. The set $D(A)$ of all double centralizers equipped with the multiplication $\left(L_{1}, R_{1}\right) \cdot\left(L_{2}, R_{2}\right)=\left(L_{1} L_{2}, R_{1} R_{2}\right)$ is an algebra.

A mapping $T: A \longrightarrow A$ is said to be a multiplier if $a T(b)=T(a) b$ for all $a, b \in A$. Clearly, if $A_{l}(A)=\{0\}\left(A_{r}(A)=\{0\}\right.$, respectively) then $T$ is a left (right) centralizer. For all $a, b \in A$, we put $a^{0}-b^{0}=0, a^{0} b=b$. We establish the Hyers-Ulam stability of double centralizers as follows:

Theorem 2.2. Let $f_{i}: A \rightarrow A$ be mappings with $f_{i}(0)=0(i=0,1)$, and let $\varphi: A^{6} \rightarrow[0, \infty)$ be a function such that

$$
\begin{gathered}
\| f_{i}(\mu x+y+z w)-\mu f_{i}(x)-f_{i}(y)-\left[(1-i)\left(f_{i}(z) w\right)^{1-i}+i\left(z f_{i}(w)\right)^{i}\right] \\
-s f_{0}(t)+f_{1}(s) t \| \leq \varphi(x, y, z, w, t, s)
\end{gathered}
$$

for all $\mu \in \mathbb{T}=\{\lambda \in \mathbb{C}:|\lambda|=1\}$ and all $x, y, z, w, s, t \in A, i=0,1$. If there exists a constant $K \in(0,1)$ such that

$$
\varphi(2 x, 2 y, 2 z, 2 w, 2 s, 2 t) \leq 2 K \varphi(x, y, z, w, s, t)
$$


for all $x, y, z, w, s, t \in A$, then there exists a unique double centralizer $(L, R)$ on A satisfying

$$
\left\|f_{0}(x)-L(x)\right\| \leq \frac{1}{2(1-K)} \varphi(x, x, 0,0,0,0)
$$

and

$$
\left\|f_{1}(x)-R(x)\right\| \leq \frac{1}{2(1-K)} \varphi(x, x, 0,0,0,0)
$$

for all $x \in A$.

Proof. We consider the set $X=\{h: A \longrightarrow A \mid h(0)=0\}$ and introduce the generalized metric on $X$ as follows:

$d\left(h_{1}, h_{2}\right):=\inf \left\{C \in(0, \infty):\left\|h_{1}(x)-h_{2}(x)\right\| \leq C \varphi(x, x, 0,0,0,0), \quad \forall x \in A\right\}$, if there exist such constant $C$, and $d\left(h_{1}, h_{2}\right)=\infty$, otherwise. Similar to the proof of [1, Theorem 2.2], we can show that $d$ is a generalized metric on $X$ and the metric space $(X, d)$ is complete. We define a mapping $T: X \longrightarrow X$ via

$$
T h(x)=\frac{1}{2} h(2 x)
$$

for all $x \in A$. First, we show that $T$ is strictly contractive on $X$. Given $h_{1}, h_{2} \in X$, let $C \in(0, \infty)$ be an arbitrary constant with $d\left(h_{1}, h_{2}\right) \leq C$, i.e.,

$$
\left\|h_{1}(x)-h_{2}(x)\right\| \leq C \varphi(x, x, 0,0,0,0)
$$

for all $x \in A$. If we substitute $x$ in the inequality (6) by $2 x$ and make use of (2) and (5), then we have

$$
\begin{aligned}
\left\|T h_{1}(x)-T h_{2}(x)\right\| & =\frac{1}{2}\left\|h_{1}(2 x)-h_{2}(2 x)\right\| \\
& \leq \frac{1}{2} C \varphi(2 x, 2 x, 0,0,0,0) \\
& \leq C K \varphi(x, x, 0,0,0,0)
\end{aligned}
$$

for all $x \in A$. Then $d\left(T h_{1}, T h_{2}\right) \leq C K$. Hence we conclude that

$$
d\left(T h_{1}, T h_{2}\right) \leq K d\left(h_{1}, h_{2}\right)
$$

for all $h_{1}, h_{2} \in X$. Hence, $T$ is a strictly contractive mapping on $X$ with a Lipschitz constant $K$. Now, we prove that $d\left(T f_{0}, f_{0}\right)<\infty$. Putting $i=0, \mu=$ $1, x=y, z=w=t=s=0$ in (1), we obtain

$$
\left\|f_{0}(2 x)-2 f_{0}(x)\right\| \leq \varphi(x, x, 0,0,0,0)
$$


for all $x \in A$. Hence

$$
\left\|\frac{1}{2} f_{0}(2 x)-f_{0}(x)\right\| \leq \frac{1}{2} \varphi(x, x, 0,0,0,0)
$$

for all $x \in A$. It follows from (7) that $d\left(T f_{0}, f_{0}\right) \leq \frac{1}{2}$. By Theorem 2.1 , there exists a unique mapping $L: A \rightarrow A$ such that $L$ is a fixed point of $T$ and that $T^{n} f_{0} \rightarrow L$, i.e.,

$$
\lim _{n \rightarrow \infty} \frac{f_{0}\left(2^{n} x\right)}{2^{n}}=L(x)
$$

for all $x \in A$, and so

$$
d\left(f_{0}, L\right) \leq \frac{1}{1-K} d\left(T f_{0}, f_{0}\right) \leq \frac{1}{2(1-K)} .
$$

In fact, the inequality (3) is true for all $x \in A$. It follows from (2) that

$$
\lim _{n \rightarrow \infty} \frac{\varphi\left(2^{n} x, 2^{n} y, 2^{n} z, 2^{n} w, 2^{n} s, 2^{n} t\right)}{2^{n}}=0 .
$$

Now, replace $2^{n} x$ and $2^{n} y$ by $x$ and $y$ respectively, and put $i=0, z=w=$ $t=s=0$ in (1). If we divide both sides of the resulting inequality by $2^{n}$, and letting $n$ tend to infinity, then the equalities (8) and (9) imply that

$$
L(\mu x+y)=\mu L(x)+L(y)
$$

for all $x, y \in A$ and all $\mu \in \mathbb{T}$. Now assume that $\mu \in \mathbb{C}$ and $\mu=\mu_{1}+i \mu_{2}$, where $\mu_{j}(j=1,2)$ are real numbers. Let $\mu_{1}=\alpha_{1}+\beta_{1}$ such that $\alpha_{1}$ is the integer part of $\mu_{1}$ and $0 \leq \beta_{1}<1$. Easily, we can write $\beta_{1}=\frac{\beta_{1,1}+\beta_{1,2}}{2}$, where $\beta_{1,1}, \beta_{1,2} \in \mathbb{T}$. We have

$$
L\left(\mu_{1} x\right)=L\left(\alpha_{1} x+\beta_{1} x\right)=\alpha_{1} L(x)+\frac{\beta_{1,1}+\beta_{1,2}}{2} L(x)=\mu_{1} L(x) .
$$

Similarly, we have $L\left(\mu_{2} x\right)=\mu_{2} L(x)$. Thus $L$ is $\mathbb{C}$-linear. We may also show from (1) that $L(x y)=L(x) y$, and so it is a left centralizer of $A$. According to the above argument, one can show that there exists a unique mapping $R: A \rightarrow A$ which is a fixed point of $T$ such that

$$
\lim _{n \rightarrow \infty} \frac{f_{1}\left(2^{n} x\right)}{2^{n}}=R(x)
$$

for all $x \in A$. Indeed, $R$ belongs to the set $\left\{h \in X, d\left(T f_{1}, h\right)<\infty\right\}$. Also, it follows from (2) that

$$
\lim _{n \rightarrow \infty} \frac{\varphi\left(0,0,0,0,2^{n} s, 2^{n} t\right)}{2^{n}}=0
$$


for all $s, t \in A$. If we put $x=y=z=w=0$ and substitute $s$ and $t$ by $2^{n} s$ and $2^{n} t$ in (1) respectively and we divide the both sides of the obtained inequality by $4^{n}$, then we get

$$
\left\|s \frac{f_{0}\left(2^{n} t\right)}{2^{n}}-\frac{f_{1}\left(2^{n} s\right)}{2^{n}} t\right\| \leq \frac{\varphi\left(0,0,0,0,2^{n} s, 2^{n} t\right)}{4^{n}} .
$$

Passing to the limit as $n \rightarrow \infty$ and from (11), we conclude that $s L(t)=$ $R(s) t$, for all $s, t \in A$.

Corollary 2.3. Let $r \in(0,1), \theta$ be a non-negative real number and let $f_{i}$ : $A \rightarrow A$ be mappings with $f_{i}(0)=0(i=0,1)$ such that

$$
\begin{gathered}
\| f_{i}(\mu x+y+z w)-\mu f_{i}(x)-f_{i}(y)-\left[(1-i)\left(f_{i}(z) w\right)^{1-i}+i\left(z f_{i}(w)\right)^{i}\right] \\
-s f_{0}(t)+f_{1}(s) t \| \leq \theta\left(\|x\|^{r}+\|y\|^{r}+\|z\|^{r}+\|w\|^{r}+\|s\|^{r}+\|t\|^{r}\right)
\end{gathered}
$$

for all $\mu \in \mathbb{T}$ and all $x, y, z, w, r, s \in A$. Then there exists a unique double centralizer $(L, R)$ on A satisfying

$$
\left\|f_{0}(x)-L(x)\right\| \leq \frac{\theta}{2-2^{r}}\|x\|^{r}
$$

and

$$
\left\|f_{1}(x)-R(x)\right\| \leq \frac{\theta}{2-2^{r}}\|x\|^{r}
$$

for all $x, y \in A$.

Proof. The result follows immediately from Theorem 2.2 by taking

$$
\varphi(x, y, z, w, s, t)=\theta\left(\|x\|^{r}+\|y\|^{r}+\|z\|^{r}+\|w\|^{r}+\|s\|^{r}+\|t\|^{r}\right)
$$

for all $x, y, z, w, s, t \in A$ and by letting $K=2^{r-1}$.

In the following corollary, we show that if $f_{1}, f_{2}$ are additive mappings, then the superstability for the inequality (1) is valid.

Corollary 2.4. Suppose that additive mappings $f_{0}, f_{1}: A \rightarrow A$ satisfy (1) and a function $\varphi: A^{6} \rightarrow[0, \infty)$ satisfies (2). Then $\left(f_{0}, f_{1}\right)$ is a double centralizer.

Proof. Since $f_{i}$ is additive, $f_{i}(0)=0$ for $i=0,1$. On the other hand, we have $f_{i}\left(2^{n} x\right)=2^{n} f_{i}(x)$ for all $x \in A$ and $i=0,1$. By Theorem 2.2, we have $\left(f_{0}, f_{1}\right)=(L, R)$ is a double centralizer. 
Corollary 2.5. Let $p_{j}, \theta$ be positive real numbers $(1 \leq j \leq 6)$ with $\sum_{j=1}^{6} p_{j} \neq$ 1 , and let $f_{i}: A \rightarrow A$ be mappings with $f_{i}(0)=0(i=0,1)$ such that

$$
\begin{gathered}
\| f_{i}(\mu x+y+z w)-\mu f_{i}(x)-f_{i}(y)-\left[(1-i)\left(f_{i}(z) w\right)^{1-i}+i\left(z f_{i}(w)\right)^{i}\right] \\
-s f_{0}(t)+f_{1}(s) t \| \leq \theta\left(\|x\|^{p_{1}}\|y\|^{p_{2}}\|z\|^{p_{3}}\|w\|^{p_{4}}\|s\|^{p_{5}}\|t\|^{p_{6}}\right)
\end{gathered}
$$

for all $\mu \in \mathbb{T}$ and all $x, y, z, w, r, s \in A$. Then $\left(f_{0}, f_{1}\right)$ is a double centralizer.

Proof. Putting $x=y=z=w=s=t=0$ in (12), we get $f_{i}(0)=0$ for $i=0,1$. Now, if we put $x=y, z=w=s=t=0$ and $\mu=1$ in (12), then we have $f_{i}(2 x)=2 f_{i}(x)$ for all $x \in A$. It is easy to see by induction that $f_{i}\left(2^{n} x\right)=2^{n} f_{i}(x)$, and so $f_{i}(x)=\frac{f_{i}\left(2^{n} x\right)}{2^{n}}$ for all $x \in A$ and $n \in \mathbb{N}$. It follows from the proof of Theorem 2.2 that $\left(f_{0}, f_{1}\right)$ is a double centralizer on $A$.

\section{$3 \quad$ Stability of multipliers}

In this section, we investigate the Hyers-Ulam stability and the superstability of multipliers.

Theorem 3.1. Let $f: A \rightarrow A$ be a mapping with $f(0)=0$ and let $\phi: A^{4} \rightarrow$ $[0, \infty)$ be a function such that

$$
\|f(\mu x+\mu y)-\mu f(x)-\mu f(y)-f(z) w+z f(w)\| \leq \phi(x, y, z, w)
$$

for all $\mu \in \mathbb{T}$ and all $x, y, z, w \in A$. If there exists a constant $K \in(0,1)$ such that

$$
\phi(2 x, 2 y, 2 z, 2 w) \leq 2 K \phi(x, y, z, w)
$$

for all $x, y, z, w \in A$, then there exists a unique multiplier $T$ on $A$ satisfying

$$
\|f(x)-T(x)\| \leq \frac{1}{2(1-K)} \phi(x, x, 0,0)
$$

for all $x \in A$.

Proof. It follows from $\phi(2 x, 2 y, 2 z, 2 w) \leq 2 K \phi(x, y, z, w)$ that

$$
\lim _{n \rightarrow \infty} \frac{\phi\left(2^{n} x, 2^{n} y, 2^{n} z, 2^{n} w\right)}{2^{n}}=0
$$

for all $x, y, z, w \in A$. Putting $\mu=1, x=y$ and $z=w=0$ in (13), we obtain

$$
\|f(2 x)-2 f(x)\| \leq \phi(x, x, 0,0)
$$


for all $x \in A$. So

$$
\left\|\frac{1}{2} f(2 x)-f(x)\right\| \leq \frac{1}{2} \phi(x, x, 0,0)
$$

for all $x \in A$. Consider the set $X:=\{h: A \rightarrow A \mid h(0)=0\}$ and introduce the generalized metric on $X$ :

$$
d\left(h_{1}, h_{2}\right):=\inf \left\{C \in \mathbb{R}^{+}:\left\|h_{1}(x)-h_{2}(x)\right\| \leq C \phi(x, x, 0,0) \text { for all } x \in A\right\},
$$

if there exist such constant $C$, and $d\left(h_{1}, h_{2}\right)=\infty$, otherwise. It is easy to show that $(X, d)$ is complete. We define a mapping $\Phi: X \rightarrow X$ by

$$
\Phi h(x)=\frac{1}{2} h(2 x)
$$

for all $x \in A$. By the same reasoning as in the proof of Theorem 2.2, $\Phi$ is strictly contractive on $X$. It follows from (17) that

$$
d(\Phi f, f) \leq \frac{1}{2}
$$

By Theorem 2.1, $\Phi$ has a unique fixed point in the set $X_{1}:=\{h \in X$ : $d(f, h)<\infty\}$. Let $T$ be the fixed point of $\Phi$. Then $T$ is the unique mapping with

$$
T(2 x)=2 T(x)
$$

for all $x \in A$ such that there exists $C \in(0, \infty)$ such that

$$
\|T(x)-f(x)\| \leq K \phi(x, x, 0,0)
$$

for all $x \in A$. On the other hand, we have $\lim _{n \rightarrow \infty} d\left(\Phi^{n}(f), h\right)=0$. Thus

$$
\lim _{n \rightarrow \infty} \frac{1}{2^{n}} f\left(2^{n} x\right)=T(x)
$$

for all $x \in A$. Hence

$$
d(f, T) \leq \frac{1}{1-K} d(f, \Phi f) \leq \frac{1}{2(1-K)} .
$$

This implies the inequality (15). It follows from (13), (16) and (18) that

$$
\begin{aligned}
\|T(x+y)-T(x)-T(y)\| & =\lim _{n \rightarrow \infty} \frac{1}{2^{n}}\left\|f\left(2^{n}(x+y)\right)+f\left(2^{n}(x)\right)-f\left(2^{n} y\right)\right\| \\
& \leq \lim _{n \rightarrow \infty} \frac{1}{2^{n}} \phi\left(2^{n} x, 2^{n} y, 0,0\right)=0
\end{aligned}
$$


for all $x, y \in A$. So

$$
T(x+y)=T(x)+T(y)
$$

for all $x, y \in A$. Thus $T$ is Cauchy additive. Putting $y=x, z=w=0$ in (13), we have

$$
\|2 \mu f(x)-f(2 \mu x)\| \leq \phi(x, x, 0,0)
$$

for all $x \in A$. Hence

$$
\begin{aligned}
\|T(2 \mu x)-2 \mu T(x)\| & =\lim _{n \rightarrow \infty} \frac{1}{2^{n}}\left\|f\left(2 \mu 2^{n} x\right)-2 \mu f\left(2^{n} x\right)\right\| \\
& \leq \lim _{n \rightarrow \infty} \frac{1}{2^{n}} \phi\left(2^{n} x, 2^{n} x, 0,0\right)=0
\end{aligned}
$$

for all $\mu \in \mathbb{T}$ and $x \in A$. So $T(2 \mu x)=2 \mu T(x)$ for all $\mu \in \mathbb{T}$ and $x \in A$. Since $T$ is a additive map, $T(\mu x)=\mu T(x)$ for all $\mu \in \mathbb{T}$ and $x \in A$. The proof of Theorem 2.2 shows that $T$ is $\mathbb{C}$-linear. If we substitute $z$ and $w$ by $2^{n} z$ and $2^{n} w$ in (13) respectively, and put $x=y=0$ and we divide the both sides of the obtained inequality by $4^{n}$, we get

$$
\left\|z \frac{f\left(2^{n} w\right)}{2^{n}}-\frac{f\left(2^{n} z\right)}{2^{n}} w\right\| \leq \frac{\phi\left(0,0,2^{n} z, 2^{n} w\right)}{4^{n}} .
$$

Passing to the limit as $n \rightarrow \infty$ and using (16), we conclude that $z T(w)=$ $T(x) w$ for all $z, w \in A$.

Corollary 3.2. Let $r \in(0,1), \theta$ be non-negative real number and let $f: A \rightarrow A$ be a mapping with $f(0)=0$ such that

$\|f(\mu x+\mu y)-\mu f(x)-\mu f(y)-f(z) w-z f(w)\| \leq \theta\left(\|x\|^{r}+\|y\|^{r}+\|z\|^{r}+\|w\|^{r}\right)$

for all $\mu \in \mathbb{T}$ and all $x, y, z, w \in A$. Then there exists a unique multiplier $T$ on A satisfying

$$
\|f(x)-T(x)\| \leq \frac{\theta}{2-2^{r}}\|x\|^{r}
$$

for all $x \in A$.

Proof. We can deduce the desired result from Theorem 3.1 if we take

$$
\phi(x, y, z, w)=\theta\left(\|x\|^{r}+\|y\|^{r}+\|z\|^{r}+\|w\|^{r}\right)
$$

for all $x, y, z, w \in A$.

In analogy with corollaries 2.4 and 2.5, we have the following results which show that under what conditions the multipliers on Banach algebras are superstable. 
Corollary 3.3. Suppose that an additive mapping $f: A \rightarrow A$ satisfies (13) and a function $\phi: A^{4} \rightarrow[0, \infty)$ satisfies (14). Then $f$ is a multiplier on $A$.

Proof. Since $f$ is additive, $f(0)=0$. On the other hand, we have $f\left(2^{n} x\right)=$ $2^{n} f(x)$ for all $x \in A$. By Theorem 3.1, $f$ is a multiplier on $A$.

Corollary 3.4. Let $p_{j}(1 \leq j \leq 4), \theta$ be positive real numbers with $\sum_{j=1}^{4} p_{j} \neq$ 1 , and let $f: A \rightarrow A$ be a mapping such that

$$
\begin{gathered}
\|f(\mu x+\mu y)-\mu f(x)-\mu f(y)-f(z) w-z f(w)\| \\
\leq \theta\left(\|x\|^{p_{1}}\|y\|^{p_{2}}\|z\|^{p_{3}}\|w\|^{p_{4}}\right)
\end{gathered}
$$

for all $\mu \in \mathbb{T}$ and all $x, y, z, w \in A$. Then $f$ is a multiplier on $A$.

Proof. If we put $x=y=z=w=0$ in (20), we have $f(0)=0$. Again, by letting $x=y, z=w=0$ and $\mu=1$ in (20), we get $f(2 x)=2 f(x)$ for all $x \in A$. Similar to the proof of Corollary 2.5, one can obtain $f(x)=\frac{f\left(2^{n} x\right)}{2^{n}}$ for all $x \in A$ and $n \in \mathbb{N}$. Now, the proof of Theorem 3.1 shows that $f$ is a multiplier on $A$.

Acknowledgements: The authors sincerely thank the anonymous reviewer for his careful reading, constructive comments and fruitful suggestions to improve the quality of the manuscript. The first and third authors would like to thank Islamic Azad University of Garmsar for the financial support.

\section{References}

[1] A. Bodaghi, I. A. Alias and M. H. Ghahramani, Ulam stability of a quartic functional equation, Abst. Appl. Anal. Volume 2012, Art. ID 232630, 9 pages, doi:10.1155/2012/232630.

[2] A. Bodaghi, I. A. Alias and M. H. Ghahramani, Approximately cubic functional equations and cubic multipliers, J. Inequal. Appl. 2011 (2011): 53.

[3] L. Cădariu and V. Radu, Fixed points and the stability of quadratic functional equations, An. Univ. Timişoara, Ser. Mat. Inform. 41 (2003), 25-48.

[4] L. Cădariu and V. Radu, On the stability of the Cauchy functional equation: A fixed point approach, Grazer Math. Ber. 346 (2004), 43-52. 
[5] J. B. Diaz and B. Margolis, A fixed point theorem of the alternative for contractions on a generalized complete metric space, Bull. Amer. Math. Soc. 74 (1968),305-309.

[6] M. Eshaghi Gordji and A. Bodaghi, On the Hyers-Ulam-Rasias stability problem for quadratic functional equations, East. J. Approximations. 16, No. 2 (2010), 123-130.

[7] M. Eshaghi Gordji and A. Bodaghi, On the stability of quadratic double centralizers on Banach algebras, J. Comput. Anal. Appl. 13, No. 4 (2011), 724-729.

[8] M. Eshaghi Gordji, A. Bodaghi and C. Park, A fixed point approach to the stability of double Jordan centralizers and Jordan multipliers on Banach algebras, U.P.B. Sci. Bull., Series A, 73, Iss. 2 (2011), 65-73.

[9] M. Eshaghi Gordji, H. Khodaei, The fixed point method for fuzzy approximation of a functional equation associated with inner product spaces, Discrete Dynamics in Nature and Society Volume 2010, Article ID 140767, 15 pages, doi:10.1155/2010/140767.

[10] M. Eshaghi Gordji, M. B. Savadkouhi, Approximation of generalized homomorphisms in quasi-Banach algebras, Analele Univ. Ovidius Constata, Math series, Vol. 17(2), 2009, 203-214.

[11] S. Helgason, Multipliers of Banach algebras, Ann. Math. (2) 64 (1956), 240-254.

[12] G. Hochschild, Cohomology and representations of associative algebras, Duke Math. J. 14 (1947), 921-948.

[13] D. H. Hyers, On the stability of the linear functional equation, Proc. Nat. Acad. Sci. U.S.A. 27 (1941), 222-224.

[14] D. H. Hyers, G. Isac and Th. M. Rassias, Stability of Functional Equations in Several Variables, Birkhäuser, Basel, 1998.

[15] B. E. Johnson, An introduction to the theory of centralizers, Proc. London Math. Soc. 14 (1964), 299-320.

[16] T. Miura, G. Hirasawa and S. E. Takahashi, Stability of multipliers on Banach algebras, Internat. J. Math. Math. Sci. 45 (2004), 2377-2381.

[17] M. S. Moslehian, F. Rahbarnia, P. K. Sahoo, Approximate double centeralizers are exact double centeralizers, Bol. Soc. Mat. Mexicana. (3) Vol. 13 (2007). 
[18] C. Park, Fixed points and Hyers-Ulam-Rassias stability of Cauchy-Jensen functional equations in Banach algebras, Fixed Point Theory and Applications. 2007 Art. ID 50175 (2007).

[19] Th. M. Rassias, On the stability of the linear mapping in Banach spaces, Proc. Amer. Math. Soc. 72 (1978), 297-300.

[20] M. Turinici, Sequentially iterative processes and applications to Volterra functional equations, Annales Univ. Mariae-Curie Sklodowska (Sect A). 32 (1978), 127-134.

[21] S. M. Ulam, Problems in Modern Mathematics, Chapter VI, Science ed., Wiley, New York, 1940.

[22] J. K. Wang, Multipliers of commutative Banach algebras, Pacific J. Math. 11 (1961), 1131-1149.

Abasalt BODAGHI,

Department of Mathematics,

Garmsar Branch,

Islamic Azad University,

Garmsar, Iran.

Email: abasalt.bodaghi@gmail.com

Madjid ESHAGHI GORDJI,

Department of Mathematics,

Semnan University, P.O. Box 35195-363,

Semnan, Iran

Email: madjid.eshaghi@gmail.com

Kamal PAYKAN,

Department of Mathematics,

Garmsar Branch,

Islamic Azad University,

Garmsar, Iran.

Email: abasalt.bodaghi@gmail.com 